\title{
Reprocessing of radiation by multi-phase gas in Low Luminosity Accretion Flows
}

\author{
A. Celotti ${ }^{1,2}$ and M.J. Rees ${ }^{2}$ \\ ${ }^{1}$ S.I.S.S.A., via Beirut 2-4, 34014 Trieste, Italy \\ ${ }^{2}$ Institute of Astronomy, University of Cambridge, Madingley Road, Cambridge CB3 OHA
}

13 September 2018

\begin{abstract}
We discuss the role that magnetic fields in low luminosity accretion flows can play in creating and maintaining a multi-phase medium, and show that small magneticallyconfined clouds or filaments of dense cold gas can dramatically reprocess the 'primary' radiation from tori. In particular, radio emission would be suppressed by free-free absorption, and an extra (weak) component would appear at optical wavelengths.

This is expected to be a common process in various environments in the central regions of Active Galaxies, such as broad line regions, accretion disk coronae and jets.
\end{abstract}

Key words: galaxies: active - radiation processes - plasmas, magnetic field

\section{INTRODUCTION}

Observational evidence, from the optical to the $\gamma$-ray band, suggests the presence of gas with a range of physical properties in the inner regions of Active Galaxies (AGN). Hot/relativistic material co-exists with 'cold' quasi-thermal gas, on a large range of scales in these complex environments: tenuous hot plasma forms a corona above or around relatively cold and dense plasma, in the form of an accretion disk or re-processing clouds; clumps of photoionized gas, responsible for the observed narrow and broad emission lines, are embedded in a tenuous hotter medium.

The formation, confinement and survival of a multiphase medium has been considered with much detail in the case of thermal re-processing by the material which gives rise to the broad lines. Difficulties associated with the maintenance of photoionized matter in two phases which are in gas pressure equilibrium, first led to the suggestion that a more effective way of sustaining temperature and density gradients may involve magnetic fields in equipartition with other forms of energy (Rees 1987).

Magnetic fields are believed to be a common ingredient in the AGN environment and so can provide effective confinement and insulation of cold gas embedded in a much hotter ambient medium. In particular, more detailed work, focused on smaller scales, has shown that this is plausibly the case in the magneto-sphere around the central engine (typically on scales $\leq 50 R_{\mathrm{s}}$ ) as well as in relativistic jets, thanks to the combined action of the magnetic and radiation fields (Celotti, Fabian \& Rees 1992; Kuncic, Blackman \& Rees 1996; Kuncic, Celotti \& Rees 1997; Celotti et al. 1998; Celotti \& Rees 1998).

Recently, much attention has focussed on accretion flows where electron-ion coupling could be ineffective because the density is low and the kinetic temperature very high. Very hot ions then provide the dominant pressure, but radiative cooling is inefficient because the energy cannot be tapped by the (cooler) electrons (Rees et al. 1982). Accretion flows of this kind, in which thermal energy is advected inwards rather than being radiated, may be relevant to the puzzling absence of substantial radiative dissipation in large elliptical galaxies (Fabian \& Rees 1995). This has raised interest in the phenomenon and led to more detailed theoretical work and the comparison of the expected spectral signatures with data. Approximate analytical solutions as well as self-consistent numerical results for the structure and properties of the flow (re-named ADAF) have been derived (e.g. Narayan \& Yi 1994, 1995; Chen et al. 1995; Mahadevan 1997; Chen, Abramowicz \& Lasota 1997). Although the faint ADAF emission cannot be clearly disentangled from other spectral components over a significant energy range, the radiative predictions seem to be, so far, globally consistent with data (e.g. Lasota et al. 1996; Reynolds et al. 1996; Di Matteo \& Fabian 1997; Esin, McClintock \& Narayan 1997; Narayan et al. 1998). However, thanks to very recent observations of a few elliptical galaxies in the radio to $\mathrm{mm}$ frequency range, Herrnstein et al. (1998) and Di Matteo et al. (1998) have pointed out that (at least) 'standard' ADAF models might be inadequate to account for the (absence of) emission in large elliptical galaxies.

Here we suggest the possibility that within the flow some of the gas can be in the form of cold and dense clumps or filaments, confined by the magnetic field expected to thread the accreting matter and examine the effects that this might produce on the observed spectral signatures of ADAFs.

(C) 0000 RAS 


\section{THE MODEL AND ITS INGREDIENTS}

Let us consider the properties of an ADAF, according to the accretion solutions derived by Narayan \& Yi (1995) and Mahadevan (1997). The self-similarity postulated by these authors must break down near the inner boundary, but may nevertheless be an adequate approximation over a substantial range in $r$.

The expected field, assumed in quasi-equipartition with the total gas pressure, is of the order of

$B=3.7 \times 10^{2} m_{8}^{-1 / 2} \dot{m}_{-3}^{1 / 2} r_{5}^{-5 / 4} \quad \mathrm{G}$

where $m_{8}=M / 10^{8} M_{\odot}$ is the mass of the black hole, $\dot{m}_{-3}=\dot{M} / 10^{-3} \dot{M}_{\mathrm{E}}$ the accretion rate in Eddington units and $r_{5}=R / 5 R_{\mathrm{S}}$ the distance in units of Schwarzschild radii. Hereafter, the viscosity parameter and the gas to total pressure have been taken as $\alpha \simeq 0.3$ and $\beta \simeq 0.5$, respectively, though it is straightforward to scale our results to other values (the constants $c_{1}$ and $c_{3}$ of the order of unity as defined in Narayan \& Yi 1995, are taken $c_{1}=0.5$ and $c_{3}=0.3$ ).

\subsection{Dense and cold gas}

The magnetic field can trap some plasma and, as this is likely to have cooled to the Compton temperature, a density contrast as high as $\sim T_{\text {vir }} / T_{\text {Compt }}$ with respect to the external/hot phase medium can be attained. Two body processes start to become very efficient in cooling the dense trapped gas, which can thus cool still further, typically reaching temperatures of the order of $T \sim 10^{4} T_{4} \mathrm{~K}$ (see Section 3.2).

The gas pressure is limited by the balance with the total magnetic field stresses and therefore a maximum density for the cold phase, achieved for a diamagnetic clump of gas, can be as high as

$n \simeq n_{\text {hot }} T_{\text {vir }} / T \sim 2 \times 10^{16} m_{8}^{-1} \dot{m}_{-3} r_{5}^{-3 / 2} T_{4}^{-1} \quad \mathrm{~cm}^{-3}$,

where $n_{\text {hot }}$ represents the density in the hot phase.

\subsection{Geometrical structure}

The spatial distribution of the gas is clearly determined by the field structure and the net external force acting on it. This would limit the scale-height of the gas to a typical value such that the tension of the distorted field (in the perpendicular direction) balances the net effect of gravity, radiation pressure and acceleration. Typically, in presence of loops with a toroidal field component, the action of gravity leads to dimensions $h_{\mathrm{g}} \sim k T / m_{\mathrm{p}} g$. However, other forces can dominate in these environments, such as the gas inertia to dynamical acceleration and the radiation force. Despite the significant opacity (mostly free-free) of the extremely dense and cold gas, because the low ADAF luminosity we do not expect radiative acceleration to play a major role in this environment (see Section 3.2), in contrast with other situations in the central regions of AGN.

It should be stressed that, from the point of view of the large-scale dynamics, these structures of cold and dense gas can be treated as a uniform loading to the global flow, as long as the individual clouds/filaments are small enough that, dragged by the field, they are constrained to follow the same dynamics as the hot material. This requires them to be smaller than the scale-height $h_{\text {eff }}$ imposed by the effective local acceleration $a$, which may be dominated by the effect of magnetic stresses or internal random motions (see also Section 3.1). As these are difficult to quantify, we have a fiducial upper limit to the typical scale-height of the order of $h_{\mathrm{g}}$

$h_{\mathrm{eff}} \simeq 10^{6} \frac{g}{a} m_{8}^{-1} T_{4} r_{5}^{2} \quad \mathrm{~cm}$.

\section{THE SURVIVAL OF THE COLD PHASE}

Let us consider the survival of clumps of cold matter thus confined and advected with the flow.

\subsection{Diffusive processes in clouds and filaments}

At the high densities inferred, radiative (see Section 3.2) and collisional timescales are much shorter that the dynamical one, estimated as the free fall time, $t_{\mathrm{ff}} \simeq 10^{4} m_{8}^{-1 / 2} r_{5}^{3 / 2} \mathrm{~s}$, allowing the gas to reach thermal and radiative equilibrium. Also, pressure equilibrium with the surrounding medium can be easily maintained in the global dynamical timescale, for typical dimension of the cold phase structures smaller than $\lesssim 10^{10} m_{8}^{-1 / 2} T_{4}^{1 / 2} r_{5}^{3 / 2} \mathrm{~cm}$.

Although $h_{\text {eff }}$ is much smaller that the extension of the hot gas, it greatly exceeds, for the inferred field strength, the characteristic gyro-radii of particles in both phases.

Resistivity is low enough to maintain the coupling between field and gas on radiative and dynamical timescales. However, collisions of the hot particles with the dense clumped gas, can lead to effective thermal conduction. As a detailed radiative computation has shown (see Section 3.2 ), the cold gas is only marginally ionized and, under this circumstance, the dominant diffusion is due - at least in the inner ADAF regions - to interactions with neutral atoms, with a typical mean free path $\lambda \simeq 0.1 n_{16}^{-1} \mathrm{~cm}$. In a timescale $t$ the cloud depth affected by thermal conduction, according to the diffusion approximation, is of the order of $d \sim 4 \times 10^{4} n_{16}^{-1 / 2} t^{1 / 2} \mathrm{~cm}$. Furthermore, the collision frequency is so high with respect to the gyration frequency (typically $\nu_{\text {coll }} \sim 10^{2} \nu_{\text {gyr }}$ ) that even in the direction perpendicular to the field line, conduction is effective (the collisional frequency is proportionately lower on larger scales). Even when clouds are thinner than the diffusion length, radiative cooling may allow them to survive.

In a disc corona or a relativistic jet the field can be either anchored in the disk (corona) or have an ordered component (jet). A possibly severe threat to filament survival - as already mentioned - is posed in an environment where random motions of field loops and large--amplitude Alfven waves would cause violent local accelerations, which correspondingly reduce the thickness of filaments that could be carried with the flow. The acceleration would be largest on the smallest scales. Let us consider, simply as an illustration, a Kolmogorov spectrum where the velocities associated with a scale $l$ are proportional to $l^{1 / 3}$ : the associated accelerations would then depend on scale as $l^{-1 / 3}$. This means that the filaments of a given scale can less easily follow the smaller-scale eddies. On the other hand, stretching of field lines would decrease the scale of the filaments.

We draw attention to this issue, emphasizing the uncertainty in the fate of dense filaments that are advected in- 
wards with the flow towards regions of progressively stronger field nearer the hole. One can envisage an element of gas, trapped within a magnetic tube, starting off in the broad line region and moving into the denser and more extreme conditions envisaged in the ADAF. But can the cool-phase gas survive, or will it be shredded and homogenized? It is unclear whether the disordered motions associated with the magnetic viscosity would shred any filaments into structures so thin that they cannot survive the effects of conductivity and diffusion.

This is an important open question for matter which is coupled to the field lines. However, material decoupled from the field (e.g. ejecta from stars, behaving like diamagnetic clouds) could survive more easily since it could be squeezed by the surrounding field but would not necessarily follow the violent random motion associated with the ' $\alpha$-viscosity'. Therefore, irrespective of the answer to the question posed above, small clouds or filaments of magnetically-confined cool gas could still have important consequences for the ADAF spectrum, as shown in the next Section.

\subsection{Radiative signatures}

Because of the tiny transverse dimensions, the cold matter occupies a small fraction of the total volume, even though a significant amount of the total mass inflow might reside in this component if its scale-height is comparable to $h_{\mathrm{g}}\left(\simeq\left(T / T_{\text {vir }}\right) R\right)$. Nevertheless, because of the extreme high density, this matter can be very effective in absorbing (mainly through free-free) and re-processing radiation impinging on it.

Furthermore, the 3D structure of the field can allow the matter to accumulate on sheet-like surfaces or filaments in the direction perpendicular to the net force, distributing it with high covering factors. Even though the volume filling factor is small, the surface covering factor can easily be of order unity.

Because of the comparatively low radiation field in ADAFs, the gas might cool to temperatures of the order of $10^{4} \mathrm{~K}$ at $\sim 5 R_{\mathrm{S}}$, allowing a density contrast between the two phases as high as $10^{8}$. The low temperature and high density cause the trapped gas to be an efficient absorber, even more so as a significant fraction of the ADAF radiation is emitted as thermal cyclo-synchrotron at low frequencies.

Therefore, if dense filaments existed within an ADAF, with (at least at some radii) a substantial covering factor, they could modify the standard ADAF spectrum. In particular, the radio and mm bump could be absorbed by free-free (re-appearing in the optical or UV bands). [Furthermore, for sufficiently ionized gas, the cold phase plasma frequency also exceeds that of the cyclo-synchrotron peak, raising the possibility of collective absorption or reflection effects.] The actual result on the radio spectrum would depend on whether the clouds were just in the inner parts (where they would absorb only the higher-frequency sub-mm radiation) or mainly in the outer part (in which case they could absorb/reflect everything), where the gas would be largely molecular and grain could survive. We stress here that this is an interesting possible complication in confronting ADAF models with observations.

Under these circumstances, the absorbed radiation can be energetically important and the spectral effect rele-

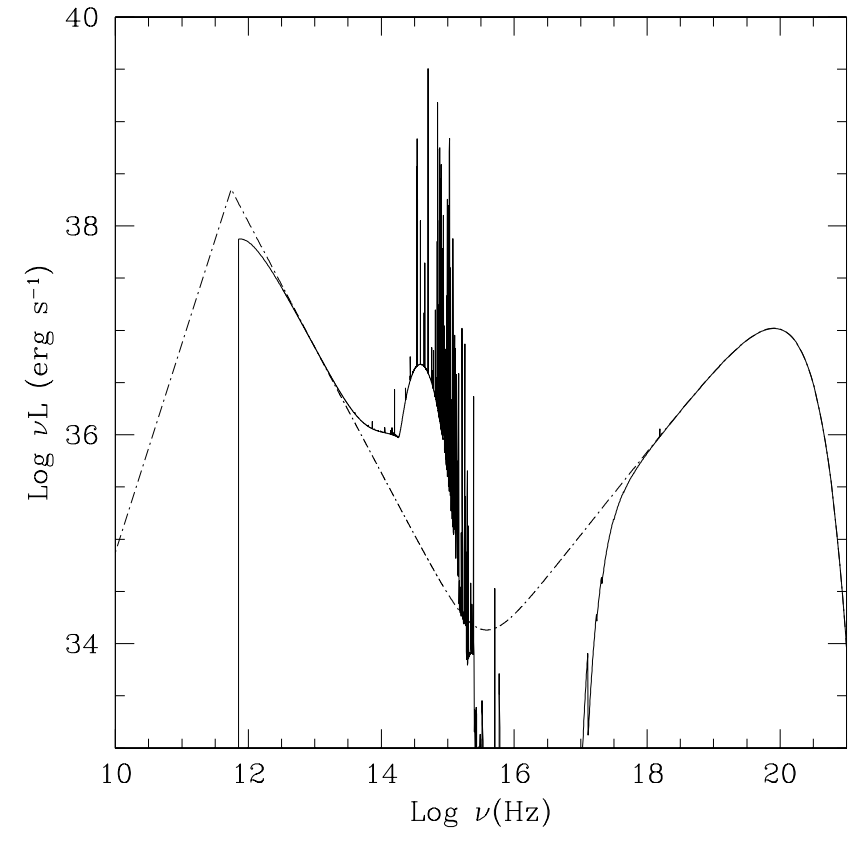

Figure 1. Reprocessed spectra emitted by cold gas structures located in an ADAF environment at about $5 R_{\mathrm{S}}$ and with a covering factor $\sim 1$. The dashed and continuous lines indicate the incident and transmitted spectra, respectively.

vant. We computed the effective temperature and conditions reached by the cold gas in thermal and radiative equilibrium, as well as the reprocessed spectrum from a detailed radiative transfer calculation, by using the code CLOUDY (it should be however noted that the code results might be not totally reliable in these extreme conditions). The local incident spectrum has been assumed to be that of an ADAF at the considered distance (e.g. Mahadevan 1997), including the synchrotron, Compton and bremsstrahlung components.

Even in the inner part of the $\mathrm{ADAF}\left(\sim 5 R_{\mathrm{S}}\right)$ the equilibrium temperature, as already mentioned, only reaches $\sim 10^{4}$ $\mathrm{K}$ and the gas is largely in neutral form.

In Fig. 1 we show the result of the computation when a covering factor of $\sim 1$ is assumed. Substantially all of the cloud heating is due to free-free absorption of the cyclosynchrotron component, which is balanced by collisional line and free-bound cooling, the longest radiative timescale being $\sim 50 \mathrm{~s}$. The spectral features in the re-processed spectrum are expected to be broadened and shifted by the high velocity motion of the gas. This includes any (weak) iron emission line, which therefore would be probably undetectable. The column density in neutral hydrogen can be less than $10^{22} \mathrm{~cm}^{-2}$, thus affecting only the soft $\mathrm{X}$-ray emission from the ADAF.

Although the ionization state and the spectral results clearly depends on the detailed incident spectrum (e.g. the amount of Comptonized synchrotron photons), the result shown here is representative of the global effect of the reprocessing by the cold material. 


\section{DISCUSSION AND CONCLUSIONS}

We have stressed the key role that magnetic fields in quasiequipartition in the accretion flow of AGN can have in creating and maintaining a multi-phase medium, by confining cold, dense gas with small filling factor. This is expected to be a common process in the central regions of Active Galaxies (broad line region, accretion disk coronae, jets,...).

As mentioned in the introduction, high energy observations of AGN strongly suggest the presence of gas in different physical conditions in the very center of AGN. In particular, spectral evidence has accumulated for a component of optically thick, cold gas, as well as a tenuous hot medium, to account for the ultraviolet to $\gamma$-ray spectrum of radioquiet AGN and galactic black hole candidates. Interestingly, Krolik (1998) has recently proposed that a consistent and stable solution for accretion disc might involve the formation of clumps of dense gas -albeit warmer and optically thicker than those considered here- embedded in a hot medium and threaded by magnetic field lines.

We have shown that thermal gas trapped by the field in an ADAF, can survive in the hot environment long enough to significantly reprocess the 'primary' radiation and produce observable spectral signatures. Its predominant effect would be to largely suppress the cyclo-synchrotron component produced by the hot gas, in the radio-mm band, which has been considered, so far, an observable indication of the presence of radiative inefficient accretion phase in our Galactic Centre and in other low-luminosity galactic nuclei (and Galactic Black Hole candidates). The resulting spectrum clearly depends on the radial distribution of the amount and covering factor of the gas. There are here two countervailing effects that are hard to quantify. The pressures (and hence the maximum densities of cool gas) are higher at small radii, so a given amount of gas would cause more absorption near the centre. On the other hand, if the cool gas consists of filaments entrained in the flow, and advected inwards with it (rather than being free of magnetic flux and merely squeezed and confined by the hot-phase medium), the violent random motions associated with the magnetic viscosity may shred and disperse it before it reaches the centre: in this case the dominant effect would be due to gas at $100-1000 R_{\mathrm{S}}$.

Interestingly, the presence of cold clumps might account for the tight upper limits recently found on the radio, $\mathrm{cm}$ and sub-mm emission of large elliptical galaxies likely to host a massive black hole accreting ISM in the radiatively inefficient ADAF regime. In particular the observational constraints on the $22 \mathrm{GHz}$ emission from the region within the molecular maser disk in NGC 4258 (Herrnstein et al. 1998) have been strengthened by the very recent results of VLA and SCUBA observations of three elliptical galaxies by Di Matteo et al. (1998). The upper limits found by these authors are indeed well below the expected emission from 'standard' ADAF models: cold material in the conditions considered here could be responsible for the absorption of a significant amount of the (unobserved) ADAF cyclosynchrotron radiation.

The strong lines and edges, but especially the thermal continuum shape in the optical-UV band, expected from the reprocessing by cold material, might be soon observable, as demonstrated by the upper limits already derived from the scattered optical light in NGC 4258 (Wilkes et al. 1995).

\section{ACKNOWLEDGMENTS}

Gary Ferland is thanked, once again, for the use of CLOUDY. The Royal Society (MJR) and the Italian MURST (AC) are acknowledged for financial support.

\section{REFERENCES}

Celotti A., Rees M.J., 1998, in Theory of Black Hole Accretion Discs, M. Abramowicz, G. Björnsson, J. Pringle eds., Cambridge University Press, in press

Celotti A., Fabian A.C., Rees M.J., 1992, MNRAS, 255, 419

Celotti A., Kuncic Z., Rees M.J., Wardle J.F.C., 1998, MNRAS, 293, 288

Chen X., Abramowicz M.A., Lasota J.-P., 1997, ApJ, 476, 61

Chen X., Abramowicz M.A., Lasota J.-P., Narayan R., Yi I., 1995, ApJ, 443, L61

Di Matteo T., Fabian A.C., 1997, MNRAS, 286, L50

Di Matteo T., Fabian A.C., Rees M.J., Carilli C.L., Ivison R.J., 1998, MNRAS, submitted

Esin A.A., McClintock J.E., Narayan R., 1997, ApJ, 489, 865

Fabian A.C., Rees M.J., 1995, MNRAS, 277, L55

Herrnstein J.R., Greenhill L.J., Moran J.M., Diamond P.J., Inoue M., Nakai N., Miyoshi M., 1998, ApJ, 497, L69

Krolik J.H., 1998, ApJ, 498, L13

Kuncic Z., Blackman E., Rees M.J., 1996, MNRAS, 283, 1322

Kuncic Z., Celotti A., Rees M.J., 1997, MNRAS, 284, 717

Lasota J.-P., Abramowicz M.A., Chen X., Krolik J., Narayan R., 1996, ApJ, 462, 142

Mahadevan R., 1997, 477, 585

Narayan R., Yi I., 1994, ApJ, 428, L13

Narayan R., Yi I., 1995, ApJ, 444, 231

Narayan R., Mahadevan R., Grindlay J.E., Popham R.G., Gammie C., 1998, ApJ, 492, 554

Rees M.J., 1987, MNRAS, 228, 47

Rees M.J., Phinney E.S., Begelman M.C., Blandford R.D., 1982, Nat, 295, 17

Reynolds C.S., Di Matteo T., Fabian A.C., Hwang U., Canizares C.R., 1996, MNRAS, 283, L111

Wilkes B.J., Schmidt G., Smith P.S., Mathur S., McLeod K.K., 1995, ApJ, 455, L13

(c) 0000 RAS, MNRAS 000, 000-000 